Classification

Physics Abstracts

$76.80-76.30 \mathrm{~K}-75.20 \mathrm{H}$

\title{
Relaxation of ytterbium in palladium
}

\author{
A. Fert, P. M. Levy (*) \\ Laboratoire de Physique des Solides (**), Université Paris-Sud, 91405 Orsay Cedex, France \\ and $\mathbf{P}$. Bonville \\ D-Ph-G/PSRM, CEN Saclay, BP no 2, 91190 Gif-sur-Yvette, France
}

(Reçu le 18 septembre 1984, révisé le 13 novembre, accepté le 16 novembre 1984)

\begin{abstract}
Résumé. - Nous montrons que l'on peut expliquer de façon satisfaisante la dépendance en température de la relaxation de $\mathrm{Yb}^{3+}$ dans le palladium en tenant compte de la partie non sphérique de l'interaction Coulombienne directe entre électrons $4 \mathrm{f}$ et électrons de conduction de caractère $d$.

Abstract. - We show that a satisfactory fit to the temperature dependence of the relaxation rate of ytterbium in palladium can be obtained by including the aspherical part of the direct Coulomb interaction between $4 \mathrm{f}$ electrons and the $\mathrm{d}$ part of the conduction electrons.
\end{abstract}

The paramagnetic relaxation rate of ytterbium in palladium was recently measured by Mössbauer experiments between $0.11 \mathrm{~K}$ and $2 \mathrm{~K}$ [1]. The data was fit to a Hirst-Orbach law [2] of the form :

$$
\frac{1}{T_{1}}=C_{\mathrm{K}} T\left[1+\beta f\left(\Delta / k_{\mathrm{B}} T\right)\right]
$$

where $f(x) \equiv x /\left(\mathrm{e}^{x}-1\right)$.

$C_{\mathrm{K}}$ represents the coefficient of the Korringa relaxation taking place within the $\Gamma_{7}$ ground state and the acceleration coefficient $\beta$ represents the presence of relaxation to an excited state at an energy $\Delta$ above the ground state. To fit the rapid rise of the relaxation above the Korringa rate one found that $\beta \sim 4$ was necessary, with $\Delta=2.5 \mathrm{~K}$ for the distance to the $\Gamma_{8}$ quadruplet and $C_{\mathrm{K}}=(120 \pm 20) \mathrm{MHz} / \mathrm{K}$. Usually ytterbium in metals is anomalous because the $4 \mathrm{f}$ electrons mix with the conduction electrons and the main contribution to the exchange interaction comes from the s-f mixing. However, ytterbium in palladium is normal ; the s-f mixing is not important and the Coulomb interaction provides the principal contribution to the exchange coupling. If one considers the conventional exchange interaction $-J \mathbf{s . S}$ one finds $\beta=1$. By considering the complete exchange interaction between $4 \mathrm{f}$ electrons and conduction electrons with $\mathrm{d}$ character one was able to improve this coefficient to $\beta \sim 2[1]$.

(*) Permanent address : Department of Physics, New York University, New York, NY 10003, U.S.A.

(**) Laboratoire associé au CNRS. 
The direct Coulomb interaction between $4 \mathrm{f}$ and conduction electrons has been found to be very important in explaining the EPR and transport properties of noble metal with rare-earth impurities [3]. We will show that a satisfactory fit to the relaxation rates of ytterbium in palladium can be obtained by including the aspherical part of the direct Coulomb interaction between the $4 \mathrm{f}$ electrons and the $d$ part of the conduction electrons. This interaction contributes to the relaxation by inducing transitions primarily to the excited states; therefore it immediately increases the acceleration coefficient $\beta$. First we consider the $4 \mathrm{f}-5 \mathrm{~d}$ direct and exchange contributions to the relaxation; then at the end we incorporate the contribution from the $4 \mathrm{f}-6 \mathrm{~s}$ exchange term.

The relaxation rate for $\mathrm{Yb}^{3+}$ in $\underline{\mathrm{Pd}}$ is given as :

$$
\frac{1}{T_{1}}=2 W_{\Gamma_{7} \rightarrow \Gamma_{7}}+\sum_{j=6,8} W_{\Gamma_{7} \rightarrow \Gamma_{j}}
$$

where $W_{\Gamma_{7} \rightarrow \Gamma_{7}}$ is the transition probability within the ground state doublet and $W_{\Gamma_{7} \rightarrow \Gamma_{j}}$ the probability of going from the ground to an excited crystal field state of $\mathrm{Yb}^{3+}$, i.e. either $\Gamma_{8}$ or $\Gamma_{6}$. As the $\Gamma_{6}$ doublet presumably lies far above the other states [1], $\Gamma_{7}$ and $\Gamma_{8}$, and as the temperature is quite low, $T<2 \mathrm{~K}$, we can easily neglect the transitions to $\Gamma_{6}$ and consider only excitations to the low lying excited quartet $\Gamma_{8}$.

The transitions within the states of the $\Gamma_{7}$ doublet and between $\Gamma_{7}$ and $\Gamma_{8}$ are induced by the Coulomb interaction of the $4 \mathrm{f}$ electrons with the conduction electrons, i.e., by the $\mathrm{k}-\mathrm{f}$ Coulomb interaction [4]. The transition probability between two states of $\mathrm{Yb}^{3+}(\alpha$ and $\beta)$ has been previously derived by us and is given as [3] :

$$
W_{\alpha \rightarrow \beta}=\frac{k_{\mathrm{B}} T f\left(\Delta_{\alpha \beta} / k_{\mathrm{B}} T\right)}{8 \pi \hbar} N^{2}\left(E_{\mathrm{F}}\right) \sum_{\sigma \sigma^{\prime}} \int_{\Omega_{k}} \int_{\Omega_{k^{\prime}}}\left|\mathrm{d} T_{\alpha \mathbf{k} \sigma \rightarrow \beta \mathbf{k}^{\prime} \sigma^{\prime}}\right|^{2} \mathrm{~d} \Omega_{k} \mathrm{~d} \Omega_{k^{\prime}}
$$

where $\mathrm{d} T$ represents the first order correction to the $T$ matrix elements due to the $\mathrm{k}-\mathrm{f}$ Coulomb interaction (direct and exchange) [3], $f\left(\Delta / k_{\mathrm{B}} T\right)$ is given in equation (1), $\Delta_{\alpha \beta}$ is the energy separation between levels $\alpha$ and $\beta$, and $N\left(E_{\mathrm{F}}\right)$ is the density of states of the conduction electrons at the Fermi level per unit volume for one spin direction. The correction to the $T$ matrix is evaluated between states of the conduction electrons which take into account the admixture of ytterbium's $5 \mathrm{~d}$ states. This admixture gives the main contribution to the Coulomb scattering. By using Friedel's theory of non-magnetic virtual bound states (VBS) the matrix element is written as [4] :

$$
\mathrm{d} T_{\alpha \mathbf{k} \sigma \rightarrow \beta \mathbf{k}^{\prime} \sigma^{\prime}}=\frac{4 \mathrm{e}^{2 i \eta_{2}} \sin ^{2} \eta_{2}}{\Delta_{\mathrm{d}} N\left(E_{\mathrm{F}}\right)} \sum_{m m^{\prime}}\left\langle m^{\prime} \sigma^{\prime}\left|v_{\alpha \beta}(J)\right| m \sigma\right\rangle Y_{2 m}^{*}\left(\Omega_{k}\right) Y_{2 m^{\prime}}\left(\Omega_{k^{\prime}}\right)
$$

where $v_{\alpha \beta}$ is the matrix element of the perturbation between the states $\alpha$ and $\beta$ of ytterbium, the sums on $m$ and $m^{\prime}$ go over all five states of the $5 \mathrm{~d}$ level, $\Delta_{\mathrm{d}}$ is the half-width of the VBS and the phase shift $\eta_{2}$ is given as :

$$
\eta_{2}=\frac{\pi}{10} Z_{\mathrm{d}}
$$

where $Z_{\mathrm{d}}$ is the number of $5 \mathrm{~d}$ electrons. The matrix elements in equation (4) can be written in terms of the elements of the $4 \mathrm{f}-5 \mathrm{~d}$ Coulomb interaction. The general expression has been previously given by us [3] for $\mathrm{Yb}^{3+}\left(4 \mathrm{f}^{13}\right)$; the leading terms are given as :

$$
\begin{aligned}
v(J)=-\frac{1}{7}\left(A_{0} \mathrm{~s}\right. & \left.+6 A_{1} \mathrm{l}-15 \sqrt{7} A_{2}\left(s^{1} \times u^{2}\right)_{\mathrm{d}}^{1}\right) . \mathrm{J}- \\
& -\left(0.356 A_{3} u_{\mathrm{d}}^{2}-0.208 A_{4}\left(s^{1} \times u^{1}\right)_{\mathrm{d}}^{2}+0.075 A_{5}\left(s^{1} \times u^{3}\right)_{\mathrm{d}}^{2}\right) \cdot \mathcal{O}^{2}(J)+\cdots
\end{aligned}
$$


where the dots represent higher-rank interactions. These higher rank terms have not been taken into account in previous analyses, and will not be considered in our analysis. The irreducible tensors $u_{\mathrm{d}}^{k}$ have unit reduced matrix elements (see Ref. [3]). The coefficients $A_{k}$ are related to the Slater direct and exchange integrals and are given in tables V and VIII of reference [3].

By placing the perturbation $v(J)$ (Eq. (6)) in the expression for the transition probability (Eqs. (3) and (4)) and by performing the angular integrations over $\mathbf{k}$ and $\mathbf{k}^{\prime}$ and the intermediate spin states $\sigma^{\prime}$, we find :

$$
W_{\alpha \rightarrow \beta}=4 \sin ^{2} \eta_{2} \frac{k_{\mathrm{B}} T}{h \Delta_{\mathrm{d}}^{2}} f\left(\Delta_{\alpha \beta} / k_{\mathrm{B}} T\right) \operatorname{Tr}\left(v_{\alpha \beta}^{*} v_{\alpha \beta}\right)
$$

where we used $h=2 \pi \hbar$ and the trace represents a sum over all ten 5 d states, i.e.,

$$
\operatorname{Tr}\left(v_{\alpha \beta}^{*} v_{\alpha \beta}\right) \equiv \sum_{m=-2}^{2} \sum_{\sigma}\left\langle m \sigma\left|v_{\alpha \beta}^{*} v_{\alpha \beta}\right| m \sigma\right\rangle
$$

Only spherical invariants of the $5 \mathrm{~d}$ operators have a non-zero trace and contribute. These traces over the products of the angular momentum and irreducible tensor operators $u^{k}$ of the $5 \mathrm{~d}$ electrons entering equation (7) have been evaluated by repeatedly using the relation for recoupling four angular momenta [5] :

$$
\left(a^{k_{1}} \times b^{k_{2}}\right)^{k} \cdot\left(a^{k_{1}} \times b^{k_{2}}\right)^{k}=\frac{2 k+1}{\left(2 k_{1}+1\right)\left(2 k_{2}+1\right)} a^{k_{1}} \cdot a^{k_{1}} b^{k_{2}} \cdot b^{k_{2}}+\cdots
$$

where the terms on the right hand side which are traceless, i.e. non-scalar, have not been enumerated. To arrive at this relation we used the definition of the scalar product of two tensor operators :

$$
a^{k} \cdot b^{k}=\sum_{m}(-)^{m} a_{m}^{k} b_{-m}^{k}
$$

which is related to the irreducible tensor product of rank zero by :

$$
a^{k} \cdot b^{k} \equiv \sqrt{2 k+1}\left(a^{k} \times b^{k}\right)^{0}
$$

By evaluating the traces entering equation (7) we find :

$$
\begin{aligned}
W_{\alpha \rightarrow \beta}=4 \sin ^{4} \eta_{2} \frac{k_{\mathrm{B}} T}{h \Delta_{\mathrm{d}}^{2}} & \left\{\frac{1}{49}\left(\frac{5}{2} A_{0}^{2}+720 A_{1}^{2}+\frac{315}{2} A_{2}^{2}\right)\left\langle\mathbf{J} . W_{\alpha \beta} \mathbf{J}\right\rangle+\right. \\
+ & {\left.\left[\frac{2}{5}(0.127) A_{3}^{2}+\frac{5}{18}(0.043) A_{4}^{2}+\frac{5}{98}(0.0056) A_{5}^{2}\right]\left\langle\mathcal{O}^{2} . W_{\alpha \beta} \mathcal{O}^{2}\right\rangle\right\} }
\end{aligned}
$$

where :

$$
\left\langle\mathbf{J} . W_{\alpha \beta} \mathbf{J}\right\rangle=\langle\alpha|\mathbf{J}| \beta\rangle\langle\beta|\mathbf{J}| \alpha\rangle f\left(\Delta_{\alpha \beta} / k_{\mathrm{B}} T\right),
$$

and similarly for the operator $\mathcal{O}^{2}(J)$.

The matrix elements of the 4 f operators $J$ and $\mathcal{O}^{2}(J)$ have been evaluated for the $\Gamma_{7}$ and $\Gamma_{8}$ states of $\mathrm{Yb}^{3+}$. Within the $\Gamma_{7}$ manifold $\left(f\left(\Delta / k_{\mathrm{B}} T\right)=1\right)$ we find :

$$
\left\langle\mathbf{J} . W_{7-7} \mathbf{J}\right\rangle=1 / 2\left(g / g_{J}\right)^{2}=9 / 2
$$

and

$$
\left\langle\mathcal{O}^{2} \cdot W_{7-7} \mathcal{O}^{2}\right\rangle=0
$$


For transitions between $\Gamma_{7}$ and $\Gamma_{8}$ we find :

$$
\left\langle\mathbf{J} . W_{7-8} \mathbf{J}\right\rangle=9 f\left(\Delta / k_{\mathrm{B}} T\right)
$$

and

$$
\left\langle\mathcal{O}^{2} \cdot W_{7-8} \mathcal{O}^{2}\right\rangle=105 f\left(\Delta / k_{\mathrm{B}} T\right) .
$$

By placing these expectation values in the transition probability (Eq. (9)) and evaluating equation (2) we find the relaxation rate for $\mathrm{Yb}^{3+}$ in $\mathrm{Pd}$ can be written in the form of equation (1), where the Korringa relaxation coefficient is given by :

$$
C_{\mathrm{K}}=\frac{90}{49}\left(A_{0}^{2}+288 A_{1}^{2}+63 A_{2}^{2}\right) \frac{\sin ^{4} \eta_{2}}{\Delta_{\mathrm{d}}^{2}} \frac{k_{\mathrm{B}}}{h}
$$

and the acceleration coefficient is :

$$
\beta=1+\frac{686}{3} \frac{\left[2 / 5(0.127) A_{3}^{2}+5 / 18(0.043) A_{4}^{2}+5 / 98(0.0056) A_{5}^{2}\right]}{\left[A_{0}^{2}+288 A_{1}^{2}+63 A_{2}^{2}\right]} .
$$

When we use the atomic values for the Coulomb integrals entering $A_{k}$ (see Ref. [3]), $\Delta_{\mathrm{d}}=1 \mathrm{eV}$ for the half-width of the $5 \mathrm{~d}$ VBS and $\eta_{2}=\pi / 10$, i.e., one $5 \mathrm{~d}$ electron for ytterbium in palladium we find :

$$
C_{\mathrm{K}}=24 \mathrm{MHz} / \mathrm{K} \text { and } \beta=10.8 .
$$

If the $5 \mathrm{~d}$ state of ytterbium is split due to the cubic crystalline field of the surrounding matrix so that only the $t_{2 g}$ triplet state is occupied we must replace the trace in equation (7) by a double sum over the states of the triplet :

$$
\sum_{\substack{\tilde{m}, \tilde{m}^{\prime} \\ \sigma, \sigma^{\prime}}}\left\langle\tilde{m} \sigma\left|v_{\alpha \beta}^{*}\right| \tilde{m}^{\prime} \sigma^{\prime}\right\rangle\left\langle\tilde{m}^{\prime} \sigma^{\prime}\left|v_{\alpha \beta}\right| \tilde{m} \sigma\right\rangle .
$$

The transition probability when only the $5 \mathrm{~d}-\mathrm{T}_{2 \mathrm{~g}}$ state is occupied has been evaluated previously by us [6] and we can readily write down the equivalent expressions to equation (12) for $C_{\mathrm{K}}$ and $\beta$. They are :

$$
C_{\mathrm{K}}=\frac{54}{49}\left(A_{0}^{2}+\frac{288}{3} A_{1}^{2}+\frac{135}{3} A_{2}^{2}\right) \frac{\sin ^{4} \eta_{\mathrm{t}}}{\Delta_{\mathrm{t}}^{2}} \frac{k_{\mathrm{B}}}{h}
$$

and

$$
\beta=1+\frac{686}{300} \frac{\left[9 / 2(0.81) A_{3}^{2}+0.24 A_{4}^{2}+0.03 A_{5}^{2}-0.09 A_{4} A_{5}\right]}{\left[A_{0}^{2}+\frac{288}{3} A_{1}^{2}+\frac{135}{3} A_{2}^{2}\right]}
$$

where $\eta_{\mathrm{t}}=\frac{\pi}{6} Z_{\mathrm{d}}$. We have evaluated these expressions by using atomic values for the coefficients $A_{k}$ [3], $\Delta_{\mathrm{t}}=1 \mathrm{eV}$ for the half-width of the $5 \mathrm{~d}-\mathrm{t}_{2 \mathrm{~g}}$ VBS and $\eta_{\mathrm{t}}=\frac{\pi}{6}$, i.e., one $5 \mathrm{~d}$ electron for ytterbium in palladium, and we find :

and

$$
C_{\mathrm{K}}=81 \mathrm{MHz} / \mathrm{K}
$$

$$
\beta=9.2 \text {. }
$$


We see that both solutions predict large values of $\beta$. This is because the aspherical Coulomb scattering gives rise to relaxation only by transitions to excited states and thus contributes to the Hirst-Orbach term and does not to the Korringa one. These values of $\beta$ are independent of the phase shifts, $\eta_{2}$ or $\eta_{\mathrm{t}}$, and on the VBS width, $\Delta_{\mathrm{d}}$ or $\Delta_{\mathrm{t}}$. They depend on the relative values of the Coulomb integrals $A_{2}$ and as mentioned, above, have been calculated by using atomic values of the $A_{k}$. A reduction of the $A_{k}$ is expected in metals. However it cannot affect significantly $\beta$ because the reduction factor seems to be approximately the same for all the $A_{k}$ [3].

To go further we have now to take into account the contributions coming from other channels than $l=2$, in particular the contribution coming from the $l=0$ channel. This later contribution is expected to lower $\beta$. Therefore, we include the additional exchange between the $4 \mathrm{f}$ electrons and the s part of the conduction electrons of the form :

$$
H_{\mathrm{s}}=J_{\mathrm{fs}} N^{-1} \mathrm{~s} . \mathrm{S} \delta(\mathbf{r}) \text {. }
$$

There is no interference between the relaxation in the $l=0$ and $l=2$ channels so that $H_{\mathrm{s}}$ provides an independent contribution to the relaxation, that is :

$$
\frac{1}{T_{1}}=\left.\frac{1}{T_{1}}\right|_{\mathrm{s}}+\left.\frac{1}{T_{1}}\right|_{\mathrm{d}} .
$$

The additional relaxation due to the s character of the conduction electrons has been previously evaluated [1], and is given as :

where :

$$
\left.\frac{1}{T_{1}}\right|_{\mathrm{s}}=C_{\mathrm{K}}^{\mathrm{s}} T\left[1+f\left(\Delta / k_{\mathrm{B}} T\right)\right]
$$

$$
C_{\mathbf{K}}^{\mathrm{s}}=2 \pi^{2}\left[\frac{g}{g_{J}}\left(g_{J}-1\right)\right]^{2}\left[J_{\mathrm{fs}} N\left(E_{\mathrm{F}}\right)\right]^{2} \frac{k_{\mathrm{B}}}{h} .
$$

Note that the acceleration coefficient for this conventional exchange interaction is $\beta^{\mathrm{s}}=1$. By taking a value of $J_{\mathrm{fs}}=0.02 \mathrm{eV}$ [7], and $N\left(E_{\mathrm{F}}\right)=1.5 \mathrm{eV}^{-1}$ atom ${ }^{-1}$ [8], and the appropriate value of $g / g_{J}=3$ and $g_{J}=8 / 7$ for the ground doublet $\Gamma_{7}$ of $\mathrm{Yb}^{3+}(J=7 / 2)$ we find :

$$
C_{\mathrm{K}}^{\mathrm{s}}=68 \mathrm{MHz} / \mathrm{K} \text {. }
$$

We now combine the s electron contribution to the relaxation coming from the $t_{2 g}$ VBS (Eq. (16)). We choose the $t_{2 g}$ solution because (1) the $T_{2 g}$ state is known to be primarily occupied for rareearth in noble metals and (2) the final agreement is better with a $t_{2 g}$ VBS than with a 5d VBS. By combining equations (16), (19) and (20) we find that the relaxation rate for $\mathrm{Yb}^{3+}$ in $\mathrm{Pd}$ (Eq. (18)) is given as :

$$
\frac{1}{T_{1}}=(68+80 \varepsilon) T\left[1+\frac{68+9.2 \times 80 \varepsilon}{68+80 \varepsilon} f\left(\Delta / k_{\mathrm{B}} T\right)\right]
$$

where the parameter $\varepsilon$ has been introduced to account for the reduction of the atomic $4 \mathrm{f}-5 \mathrm{~d}$ Coulomb integrals to their effective values in metals [3]. This reduction is not necessary for the $\mathrm{s}$ electron contribution, as $J_{\mathrm{fs}}$ is already the effective coupling constant. From equation (21) we see that :

and

$$
C_{\mathrm{K}}=68+80 \varepsilon \mathrm{MHz} / \mathrm{K}
$$

$$
\beta=\frac{68+9.2 \times 80 \varepsilon}{68+80 \varepsilon} .
$$


With $\varepsilon=0.55$ we obtain $\beta=4.22 ; C_{\mathrm{K}}=112 \mathrm{MHz} / \mathrm{K}$, to be compared with the experimental values, $\beta=4.2 \pm 1$ and $C_{\mathrm{K}}=120 \mathrm{MHz} / \mathrm{K}$. The results on $\beta$ and $C_{\mathrm{K}}$ have to be discussed separately. In our model $\beta$ depends only on the weighting of the $l=0$ and $l=2$ channels, i.e. on the ratio of the parameters $J_{\mathrm{fs}}$ and $\varepsilon$. We have taken the value of $J_{\mathrm{fs}}$ estimated by Devine et al. [7] and a good agreement is then obtained with $\varepsilon=0.55$, i.e. with a reduction of the Coulomb integrals by a factor $\sqrt{\varepsilon}=0.74$. This value is close to what can be expected (for rare-earths in gold and silver the reduction factor of the Coulomb integrals has been estimated to be about 0.5). It thus appears that a good agreement is obtained for the acceleration factor $\beta$ by a very plausible combination of the contributions from the $l=2(\beta=9.2)$ and $l=0(\beta=1)$ channels. The good agreement obtained for $C_{\mathrm{K}}$ is less significant because $C_{\mathrm{K}}$ depends on poorly known additional parameters, the number $Z_{\mathrm{t}}$ of $\mathrm{d}$ electrons $\left(Z_{\mathrm{t}}=10 \eta_{\mathrm{t}} / \pi\right)$ and the $\mathrm{t}_{2 \mathrm{~g}}$ VBS width. We can only note that this agreement is obtained with reasonable values of these parameters. In our second solution with a $5 \mathrm{~d}$ VBS a similar agreement could be obtained only by taking too small values of $\Delta_{\mathrm{d}}$.

In summary by assuming that the relaxation of ytterbium in palladium by the conduction electrons takes place through the $4 \mathrm{f}-5 \mathrm{~d}$ and $4 \mathrm{f}-6 \mathrm{~s}$ Coulomb interactions we are able to fit the data on the relaxation rate at low temperatures. The $4 \mathrm{f}-5 \mathrm{~d}$ interaction has the quadrupolar term which couples the ground doublet $\Gamma_{7}$ to the excited states $\Gamma_{8}$ without creating any relaxation within the doublet. Therefore we find the appropriate acceleration coefficient $\beta$ which describes the rapid rise of the relaxation above the Korringa rate given by $C_{\mathrm{K}} T$. This large $\beta$ was missing in the previous analyses of reference [1]. However we know that a new calculation taking into account the aspherical Coulomb scattering in the formalism of [1] is now in progress [9].

\section{Acknowledgments.}

We would like to thank B. Coqblin and L. C. Lopes for very useful discussions. This work was supported in part by the National Science Foundation under grant DMR 81-20673, and under the United States-France program of scientific cooperation through grant INT 82-12503. One of us (P.M.L.) gratefully acknowledges the hospitality of the Laboratoire de Physique des Solides of the Université de Paris-Sud during his stay in France.

\section{References}

[1] Bonville, P., Gonzalez-Jimenez, F., Imbert, P., Jéhanno, G., Lopes, L. C., Battacharjee, A. K. and CoQblin, B., J. Physique 45 (1984) 467.

[2] HiRst, L. L., Phys. Rev. 181 (1969) 597.

[3] Lacueva, G., Levy, P. M. and Fert, A., Phys. Rev. B 26 (1982) 1099.

[4] Fert, A. and Levy, P. M., Phys. Rev. B 16 (1977) 5052.

[5] FANO, U. and RACCAH, G., Irreducible Tensorial Sets (Academic Press, New York) 1959, p. 56.

[6] See reference [2], equation $(3,12)$ and set $\eta_{1 / 2}=\eta_{3 / 2}$ as we assume there is no spin-orbit splitting of the $t_{2 \mathrm{~g}}$ level for ytterbium in palladium.

[7] Devine, R. A. B., ZingG, W. and Moret, J. M., Solid State Commun. 11 (1972) 233. They find values of $\left(J_{\mathrm{fs}}\right)$ between $0.014 \mathrm{eV}$ and $0.023 \mathrm{eV}$.

[8] Dye, D. H., Campbell, S. A., Crabtree, G. W., Kettersen, J. B., Sandresara, N. B., Vuillemain, J. J., Phys. Rev. B 23 (1981) 462.

[9] Lopes, L. C., Battacharjee, A. K., Coqblin, B. and Bonville, P., unpublished. 